\title{
Pulmonary papillary squamous cell carcinoma: a population-based analysis of incidence, treatment, and prognosis
}

\author{
Qingchen Yuan ${ }^{1,2 \#}, \mathrm{Na} \mathrm{Sun}^{3 \#}$, Zibo Meng ${ }^{4}, \mathrm{Xiao} \mathrm{Chen}^{1,2}$ \\ ${ }^{1}$ Department of Cardiology, Union Hospital, ${ }^{2}$ Key Lab of Molecular Biological Targeted Therapies of the Ministry of Education, Union Hospital, \\ ${ }^{3}$ Department of Pathogen Biology, School of Basic Medicine, ${ }^{4}$ Department of Pancreatic Surgery, Union Hospital, Tongji Medical College, \\ Huazhong University of Science and Technology, Wuhan 430022, China \\ Contributions: (I) Conception and design: Q Yuan, N Sun; (II) Administrative support: X Chen; (III) Provision of study materials or patients: Z Meng; \\ (IV) Collection and assembly of data: Q Yuan, N Sun; (V) Data analysis and interpretation: Q Yuan, Z Meng; (VI) Manuscript writing: All authors; (VII) \\ Final approval of manuscript: All authors. \\ "These authors contributed equally to this work. \\ Correspondence to: Xiao Chen. Department of Cardiology, Union Hospital, Tongji Medical College, Huazhong University of Science and Technology, \\ Wuhan 430022, China. Email: skycreeper@126.com; Zibo Meng. Department of Pancreatic Surgery, Union Hospital, Tongji Medical College, \\ Huazhong University of Science and Technology, Wuhan 430022, China. Email: zibomeng@hust.edu.cn; 343368187@qq.com.
}

\begin{abstract}
Background: Pulmonary papillary squamous cell carcinoma (PSCC) is a rare variant of pulmonary squamous cell carcinoma (SCC). This study aims to improve relevant understanding and demonstrate the incidence, treatment, and prognosis of pulmonary PSCC using a population-based database.

Methods: The Surveillance, Epidemiology and End Results (SEER) database was used to extract data of cases diagnosed with PSCC from 1973 to 2015 and analyze the incidence, treatment, and prognosis.

Results: A total of 151 pulmonary PSCC cases were identified. The incidence of pulmonary PSCC cases in 2015 was 0.009 per 100,000 persons. The tumor grade of pulmonary PSCC cases was significantly lower than that of SCC cases $(\mathrm{P}<0.001)$. Both cancer-specific (CSS) and overall survival (OS) of pulmonary PSCC were significantly higher than those of other pulmonary SCCs $(\mathrm{P}<0.001)$. The outcomes of pulmonary PSCC cases receiving surgery were significantly better than cases without surgery $(\mathrm{P}<0.001)$. On the contrary, patients with radiotherapy had a worsened prognosis compared with those without radiotherapy $(\mathrm{P}<0.05)$. As expected, the prognosis of pulmonary PSCC cases receiving surgery was significantly better than that of pulmonary PSCC cases receiving chemotherapy and radiotherapy (both $\mathrm{P}<0.05$ ).

Conclusions: Our population-based evidence shows that pulmonary PSCC, as a rare cancer, has a better prognosis compared with other pulmonary SCCs. Surgery was the only effective treatment to improve CSS and OS, while chemotherapy was ineffective and radiotherapy worsened prognosis.
\end{abstract}

Keywords: Pulmonary cancer; papillary squamous cell carcinoma (PSCC); survival analysis

Submitted Jul 06, 2019. Accepted for publication Sep 12, 2019.

doi: $10.21037 /$ jtd.2019.09.71

View this article at: http://dx.doi.org/10.21037/jtd.2019.09.71

\section{Introduction}

Pulmonary malignancies have put large social and economic burdens on human society. Currently, pulmonary squamous cell carcinoma (SCC) is the second most prevalent pulmonary malignancy (1). However, as a subtype of pulmonary SCC, pulmonary papillary SCC (PSCC) has a low incidence rate $(2,3)$. Principal symptoms of pulmonary
PSCC include cough, hemoptysis, and pulmonary infection, but asymptomatic patients also exist (4). Apart from radioactive examination such as computed tomography (CT) scanning, bronchoscopy biopsy allows for precise diagnosis of pulmonary PSCC (5). In addition, magnetic resonance imaging and positron emission tomography-CT (PET-CT) are also able to detect clinically invisible PSCC at an early 
stage (6).

Compared with other subtypes of pulmonary SCC, pulmonary PSCC possesses distinctively different morphological properties: a well differentiated SCC featuring a papillary/exophytic growth pattern (7). Grossly, the sessile lesion can be either single or multiple wellencompassed masses enlarging and obstructing the airways. Microscopically, the fingerlike structure with neoplastic epithelium and a fibrovascular core is remarkable. However, while pulmonary PSCC in situ has an intact basal membrane under epithelial proliferation, its invasive type manifests epithelial infiltration to the stromal core or beyond. Therefore, such uncertain (benign/malignant) properties of pulmonary PSCC may influence treatment efficacy and patient survival.

Despite sporadic reports on pulmonary PSCC $(5,8)$, there have been no population-based analyses of pulmonary PSCC. Hence, this study examines the Surveillance, Epidemiology and End Results (SEER) registry to evaluate the incidence, treatment, and prognosis of pulmonary PSCC. We analyzed clinical and pathological features of pulmonary PSCC and compared the prognosis of various treatments, including surgery, chemotherapy, and radiotherapy. By evaluating such population-based evidence, we hope to improve the relevant understandings of the underexplored pulmonary PSCC.

\section{Methods}

\section{Patient and tumor characteristics}

Data extraction of pulmonary PSCC and other pulmonary SCC cases from 1973 to 2015, including frequency, incidence, and survival data, was executed in the SEER 18 database. Because of the omitted patient identifiers, it was unnecessary to obtain the approval of the Institutional Review Board in this study.

Based on the International Classification of Disease for Oncology, 3rd Edition (ICD-O-3), pulmonary PSCC was identified by the histology code 8052 and pulmonary SCCs by codes $8070-8076,8078,8083,8084$, and 8094 . We used behavior code 3 to identify malignant tumors. After filtration, only cases labeled with ICD-O-3 topography codes including the primary site of the lung and bronchus: C34.0 (main bronchus), C34.1 (upper lobe, lung), C34.2 (middle lobe, lung), C34.3 (lower lobe, lung), C34.8 (overlapping lesion of lung), and C34.9 [lung, not otherwise specified (NOS)] were selected for subsequent analysis.
Patients with diagnostic confirmation (microscopically/ not microscopically confirmed) were included for further analysis and those with unknown diagnostic confirmation were excluded. Classification and statistical analysis were performed based on patient profiles including sex, ethnicity, marital status, age, tumor location, and grade. Tumor characteristics were depicted by recording the TumorNode-Metastasis (TNM) stage, American Joint Committee on Cancer (AJCC) stage, and intervention type. Patients who survived to the deadline, lost to follow-up, or died of other reasons belong to the right-censored data for the overall survival (OS) and cancer-specific survival (CSS) analysis.

\section{Incidence and survival}

All rates were reported per 100,000 persons and age of the patients was adapted for the 2000 US Standard Population (19 age groups, census P25-1130). The annual percent change (APC) in the incidence rate was also demonstrated using 1-year endpoints to analyze survival rate. Two parameters, OS and CSS, were used to reflect survival trends and calculated in the Kaplan-Meier model.

\section{Statistical analysis}

Statistical analysis was performed using SPSS (version 20.0, SPSS Inc, Chicago, IL, USA) or SEER*Stat 8.3.5 software (National Cancer Institute, Bethesda, Maryland) following the extraction of frequency, incidence, and survival data in all cases from the SEER 18 database. Incidence data were analyzed using weighted least squares to generate the annual percentage change based on 1-year endpoints. Student's $t$-tests and $\chi$-squared tests were used for continuous and categorical variables, respectively. All $\mathrm{P}$ values were two-tailed and those $<0.05$ were considered statistically significant.

\section{Results}

\section{Patient characteristics}

In the SEER 18 database, 151 patients with pulmonary PSCC and 197,415 patients with other pulmonary SCCs were identified from 1973 to 2015. All demographic characteristics are shown in Table 1. Patients with pulmonary PSCC were significantly younger than patients with other pulmonary SCCs (median: 66 vs. 69 years, mean: $65.77 v s$. 
Table 1 Patient characteristics for pulmonary PSCC and other types of pulmonary SCC

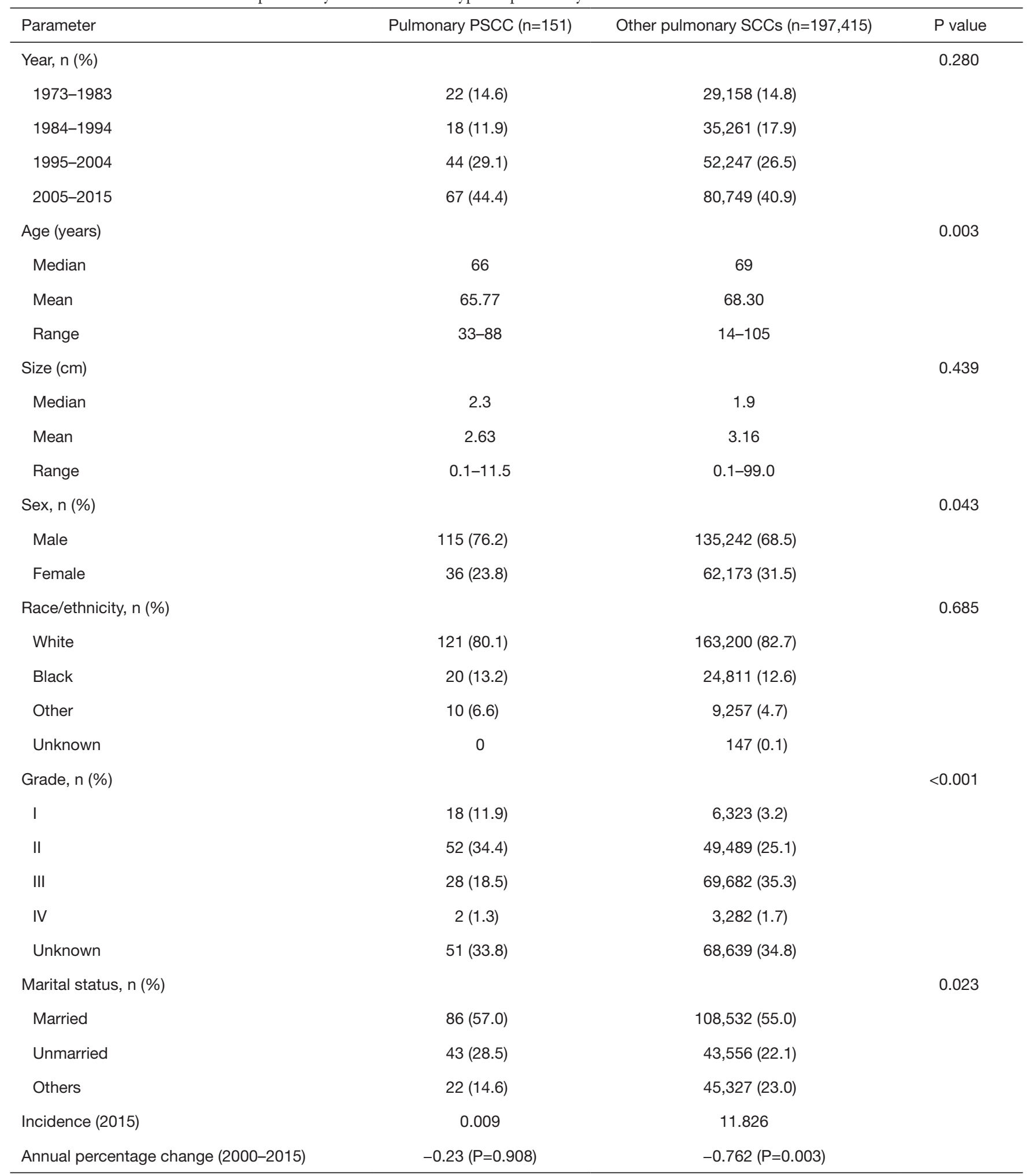

Rates are per 100,000 and age adjusted to the 2000 US Standard Population (19 age groups, census P25-1130) standard. PSCC, papillary squamous cell carcinoma; SCC, squamous cell carcinoma. 
Table 2 TNM stage for pulmonary PSCC (AJCC Stage $7^{\text {th }}$, year 2010+, n=44)

\begin{tabular}{|c|c|c|}
\hline Stage & Number & Percent \\
\hline \multicolumn{3}{|l|}{ T stage } \\
\hline $\mathrm{T} 1$ & 9 & 20.5 \\
\hline T2 & 17 & 38.6 \\
\hline T3 & 11 & 25.0 \\
\hline $\mathrm{T} 4$ & 5 & 11.4 \\
\hline $\mathrm{TX}$ & 2 & 4.5 \\
\hline \multicolumn{3}{|l|}{$\mathrm{N}$ stage } \\
\hline No & 22 & 50.0 \\
\hline N1 & 8 & 18.2 \\
\hline N2 & 11 & 25.0 \\
\hline NX & 3 & 6.8 \\
\hline \multicolumn{3}{|l|}{ M stage } \\
\hline MO & 33 & 75.0 \\
\hline M1 & 11 & 25.0 \\
\hline \multicolumn{3}{|l|}{ AJCC stage } \\
\hline I & 11 & 25.0 \\
\hline II & 10 & 22.7 \\
\hline III & 10 & 22.7 \\
\hline IV & 11 & 25.0 \\
\hline Unknown & 2 & 4.5 \\
\hline
\end{tabular}

TNM, Tumor-Node-Metastasis; PSCC, papillary squamous cell carcinoma; AJCC, American Joint Committee on Cancer.

68.30 years; $\mathrm{P}=0.003)$. Compared with other pulmonary SCCs, pulmonary PSCC was comprised of a significantly larger proportion of males $(76.2 \%$ vs. $68.5 \% ; \mathrm{P}=0.043)$ and married patients $(57.0 \%$ vs. $55.0 \%$; $\mathrm{P}=0.023)$. Patients with pulmonary PSCC had lower grade lesions than those of other pulmonary SCCs $(\mathrm{P}<0.001)$. No difference existed regarding the tumor size and race/ethnicity $(\mathrm{P}=0.439$ and 0.685 , respectively).

\section{Tumor characteristics}

For pulmonary PSCC, the TNM and AJCC stages are presented in Table 2. In pulmonary PSCC, the T2 stage was the most identified (38.6\%), followed by T3 (25.0\%), T1 (20.5\%), and T4 (11.4\%). Lymph node involvement was moderate with $50 \%$ N0. For distant metastasis, metastasis (M1) appeared in $25.0 \%$ of cases and $75.0 \%$ had no distant metastasis. For AJCC stage, the proportion of stage I was equal to that of stage IV accounting for $25.0 \%$, while both stage II and III reached $22.7 \%$.

\section{Incidence analysis}

The incidence of pulmonary PSCC was verified to be 0.009 per 100,000 persons between 2000 and 2015, after age adjustment to the 2000 US Standard Population (19 age groups, census P25-1130; Table 1). Although annual percentage change (2000-2015) was $-0.23 \%$, this change was not significant $(\mathrm{P}=0.908$; Table 1, Figure 1). In contrast, the incidence of other pulmonary SCCs was 11.826 per 100,000 persons, which exhibited a significant annual percentage change (2000-2015) of $-0.762 \%(\mathrm{P}=0.003$; Table 1, Figure 1).

\section{Treatment and survival analysis}

The profiles of treatment type are summarized in Table 3. Specifically, surgery was executed in $69.5 \%$ and $54.8 \%$ of patients with pulmonary PSCC and other pulmonary SCCs, respectively $(\mathrm{P}<0.001)$. Among different surgical options, partial pneumonectomy was the most common clarified surgery type in pulmonary PSCC $(21.2 \%)$ and other pulmonary SCCs (11.6\%). Radiotherapy was significantly less used in pulmonary PSCC (35.8\%) compared with other SCCs $(49.2 \% ; \mathrm{P}=0.001)$. Chemotherapy was used in $21.9 \%$ and $69.8 \%$ of patients with pulmonary PSCC and other pulmonary SCCs, respectively $(\mathrm{P}=0.025)$. Furthermore, in 44 pulmonary PSCC patients with TNM stage, those receiving radiotherapy or chemotherapy had a higher AJCC stage ( $\mathrm{P}=0.009$ and 0.036 , respectively). Additionally, pulmonary PSCC patients receiving chemotherapy had a significantly higher $\mathrm{N}$ stage $(\mathrm{P}=0.033)$.

For both CSS and OS, pulmonary PSCC demonstrated a significantly better prognosis than other pulmonary SCC (Figure 2). We used survival rate to evaluate the effectiveness of surgery, chemotherapy, and radiotherapy for pulmonary PSCC treatment. Kaplan-Meier analysis on OS and CSS showed significant improvement after surgery (Figure $3 A, B$ ). Meanwhile, as chemotherapy had no beneficial effect 

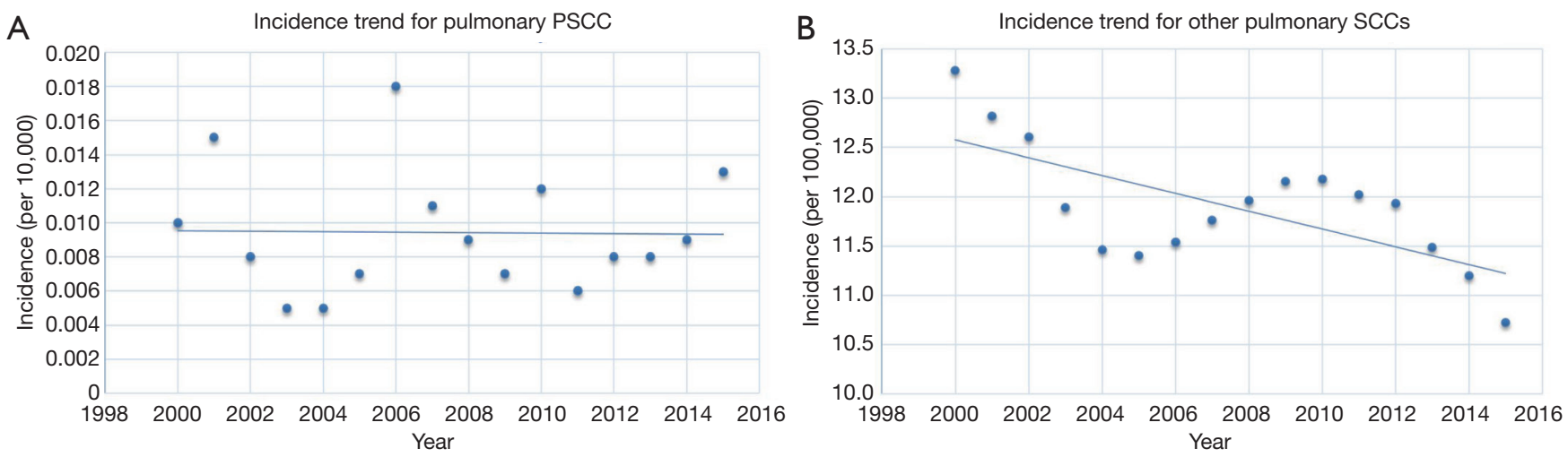

Figure 1 Incidence trends for (A) pulmonary papillary squamous cell carcinoma (PSCC) and (B) other types of pulmonary squamous cell carcinoma (SCC).

Table 3 Treatments for pulmonary PSCC and other types of pulmonary SCC

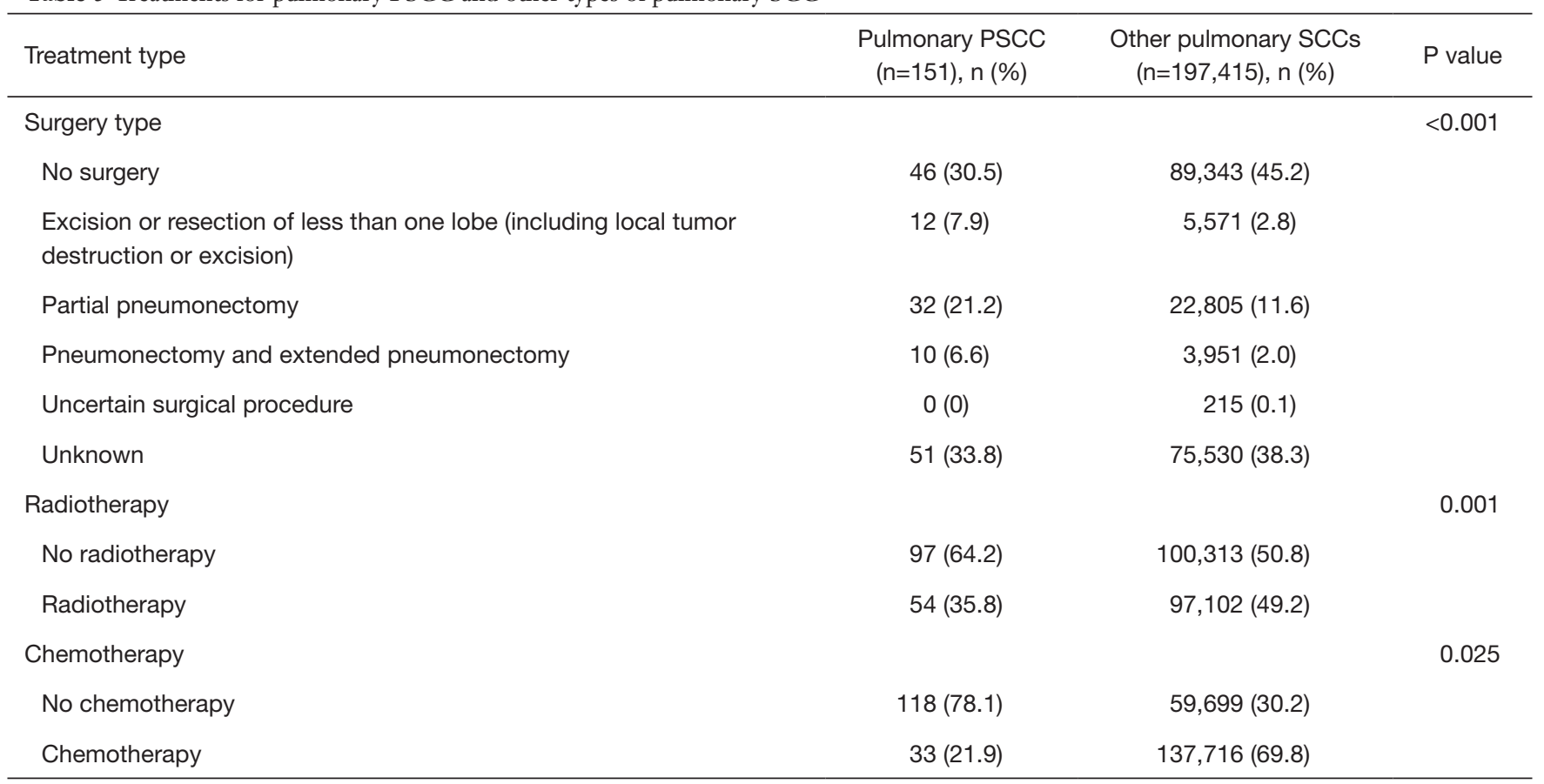

PSCC, papillary squamous cell carcinoma; SCC, squamous cell carcinoma.

(Figure 3C,D), radiotherapy-treated patients had a worsened prognosis (Figure 3E,F). Moreover, we made a comparison of different treatment modalities, including surgery alone, chemotherapy alone, radiotherapy alone, and combined therapy (surgery + chemotherapy; surgery + radiotherapy) and the results revealed that surgery is the most effective factor to improve the prognosis of pulmonary PSCC, compared with chemotherapy and radiotherapy (Figure 4).

\section{Discussion}

Pulmonary malignancies have been well studied $(9,10)$ and conventional SCC is a common cancer type (1,11-14). However, our understanding of common SCC types cannot be completely applied to rare variants of SCC, such as PSCC $(7,15-19)$. Pulmonary PSCC is very rare and consequently, there is a lack of research. As no populationbased studies on pulmonary PSCC have been conducted, 

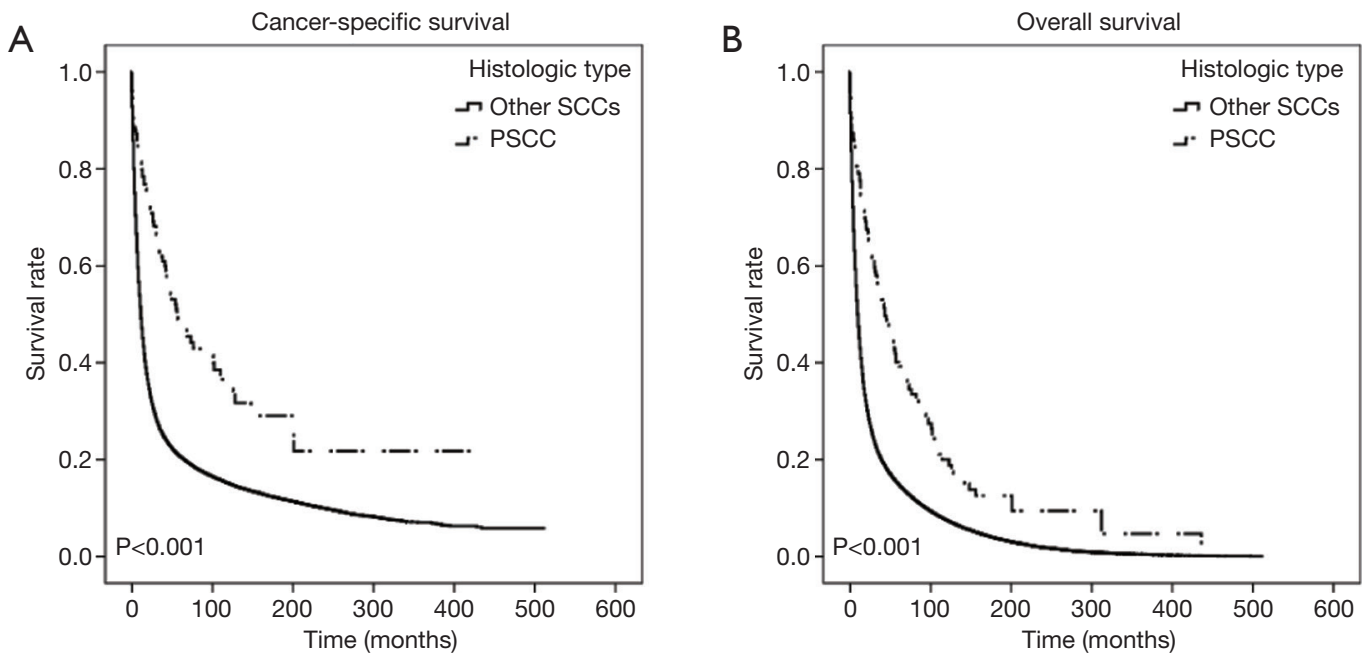

Figure 2 Survival analysis between pulmonary papillary squamous cell carcinoma (PSCC) and other types of pulmonary squamous cell carcinoma (SCC). (A) Cancer-specific survival; (B) overall survival.

this study aimed to improve the understanding of pulmonary PSCC with respect to the incidence, treatment, and prognosis by providing population-based evidence.

Pulmonary PSCC is a special variant of SCC, characterized by its histologic papillary/exophytic growth pattern consisting of a fibrovascular core covered by malignant squamous epithelium. Pulmonary PSCC differs from most common pulmonary SCCs marked by abundant intracellular and/or extracellular keratin material. Another important differential diagnosis of PSCC is squamous papilloma, a common benign tumor. Both PSCC and squamous papilloma can have exophytic growth patterns. Thus, microscopic and cytological examination is essential for differential diagnosis. PSCC contains papillae completely covered by immature basaloid cells, which are cytologically malignant. The overlying squamous epithelium is malignant and surface keratinization is usually limited or absent. However, squamous papilloma has benign squamous epithelium encompassing delicate fibrovascular cores without downward pushing growth into the lamina propria. Focal cytologic abnormalities, such as individual cell keratinization and abnormal mitosis, may also be seen but do not reach the level of dysplasia in PSCC. Therefore, it is mainly the presence of malignant epithelium that separates PSCC from squamous papilloma.

Though not investigated in this study, as the SEER database does not contain relevant data, the potential role of human papillomavirus (HPV) in the development of pulmonary PSCCs should be considered. HPV has been proven to act in wide-range cancers (20-22). In addition, among studies of PSCC on other sites, especially head and neck, HPV participates in the development of PSCC (19,23-25). Paradoxically, for certain types of SCCs, HPVpositive tumors tend to have both overall and diseasespecific survival benefits compared with HPV-negative tumors $(26,27)$. Unfortunately, to date, no adequate evidence allows us to depict a clear picture of HPV in pulmonary PSCC.

In our study, for tumor grade, most pulmonary PSCCs were initially recognized at grade II which is significantly lower than other SCCs, in which grade III accounts for the largest proportion (Table 1). Meanwhile, the proportion of patients without surgery significantly differed between pulmonary PSCC (30.5\%) and other pulmonary SCCs (45.2\%; Table 3). Such differences can be attributed to the timely detection of most pulmonary PSCCs; in this way, the opportunity for surgical intervention is still possible. In addition, the papillary/exophytic growth pattern of pulmonary PSCC to the airway may discomfort patients at an early stage and thus, be another factor contributing to timely treatment. However, TNM stage was only available in more than a quarter $(n=44)$ of cases. Although only some cases reported TNM stage, there was enough evidence indicating the low invasiveness of PSCC $(28,29)$. To our surprise, in this study, situations based on the TNM stage were not comparatively optimistic. The number of early staged pulmonary PSCCs was equal to late staged lesions: stage I and stage IV are the most commonly described, both 

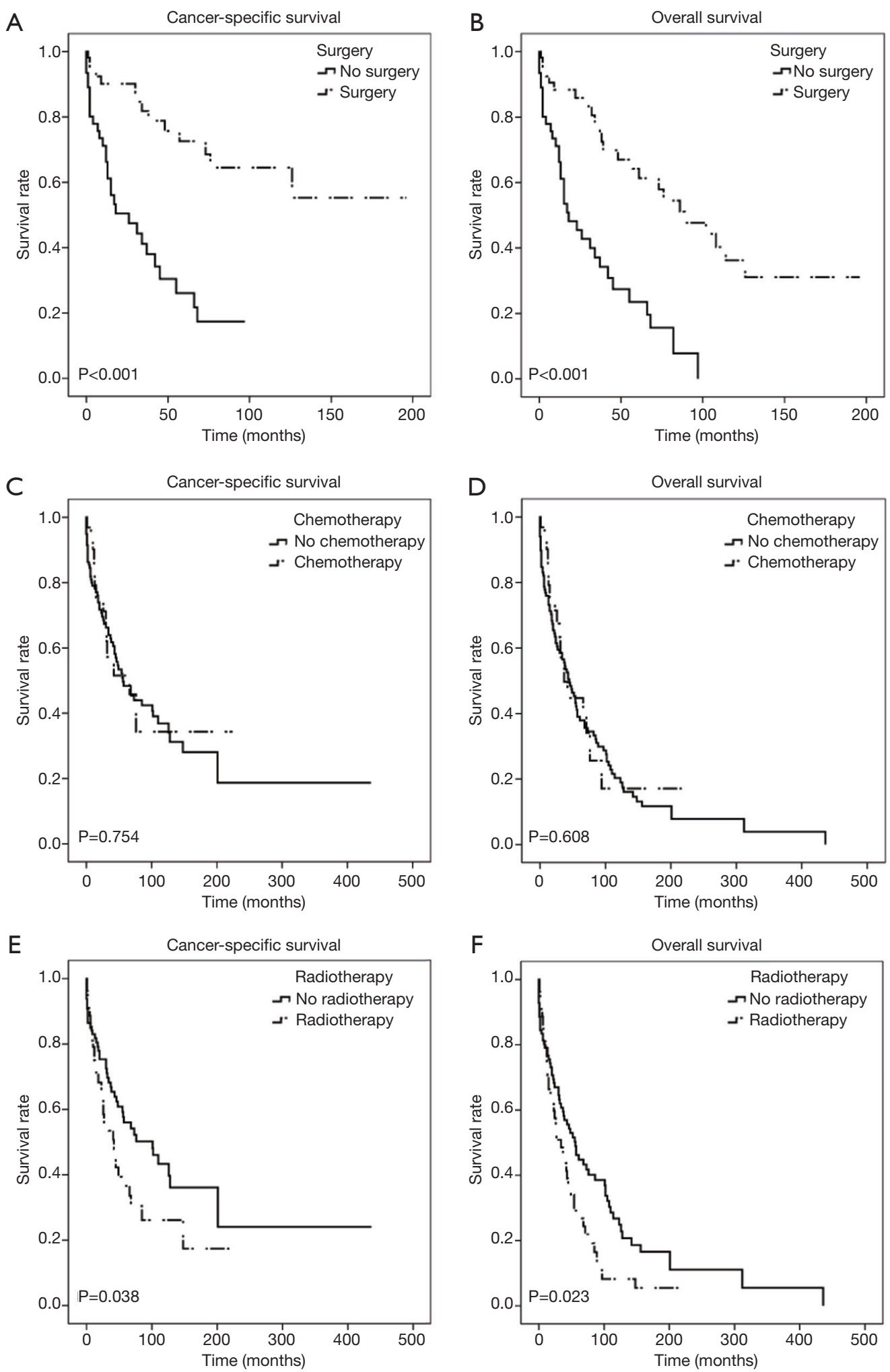

Figure 3 The effect of different treatments on the prognosis of patients with pulmonary papillary squamous cell carcinoma (PSCC). Cancerspecific survival: (A) no surgery $v$ s. surgery; (C) no chemotherapy $v$ s. chemotherapy; and (E) no radiotherapy vs. radiotherapy. Overall survival: (B) no surgery vs. surgery; (D) no chemotherapy $v s$. chemotherapy; and (F) no radiotherapy vs. radiotherapy. 
A
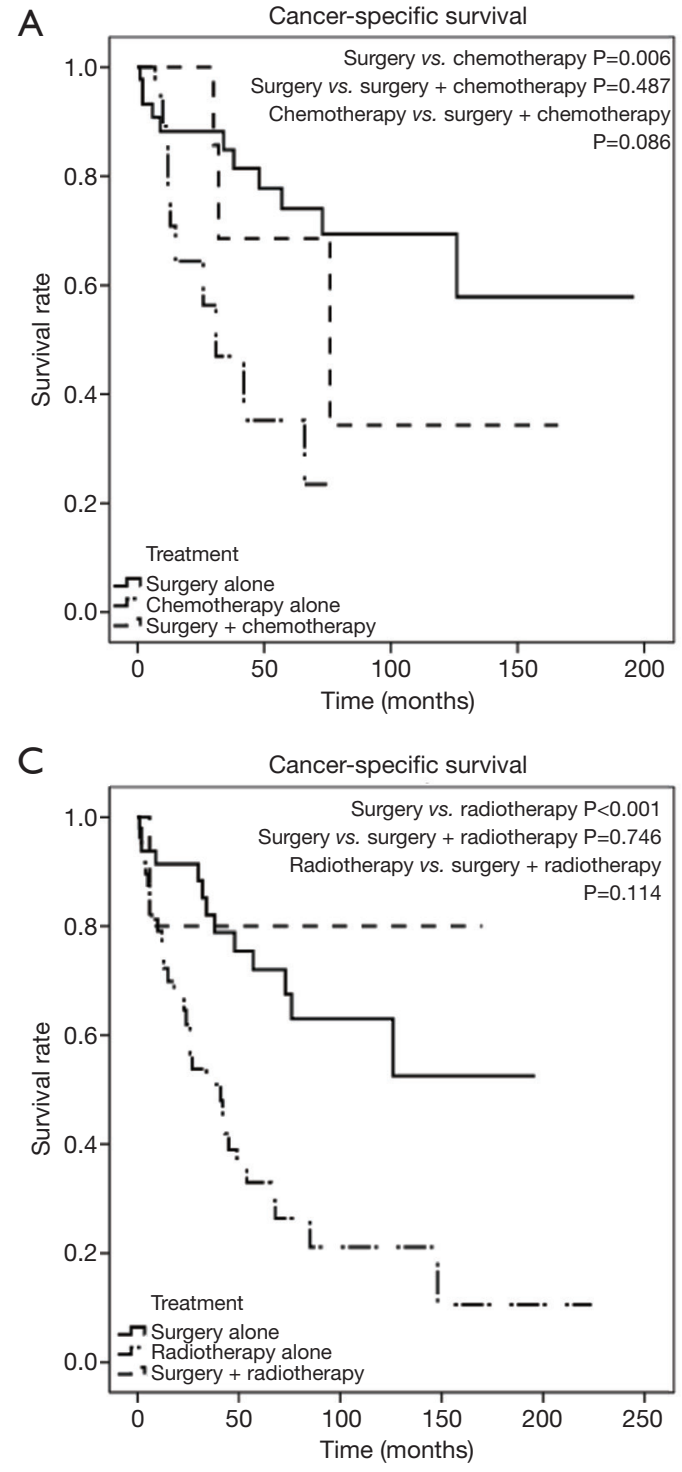
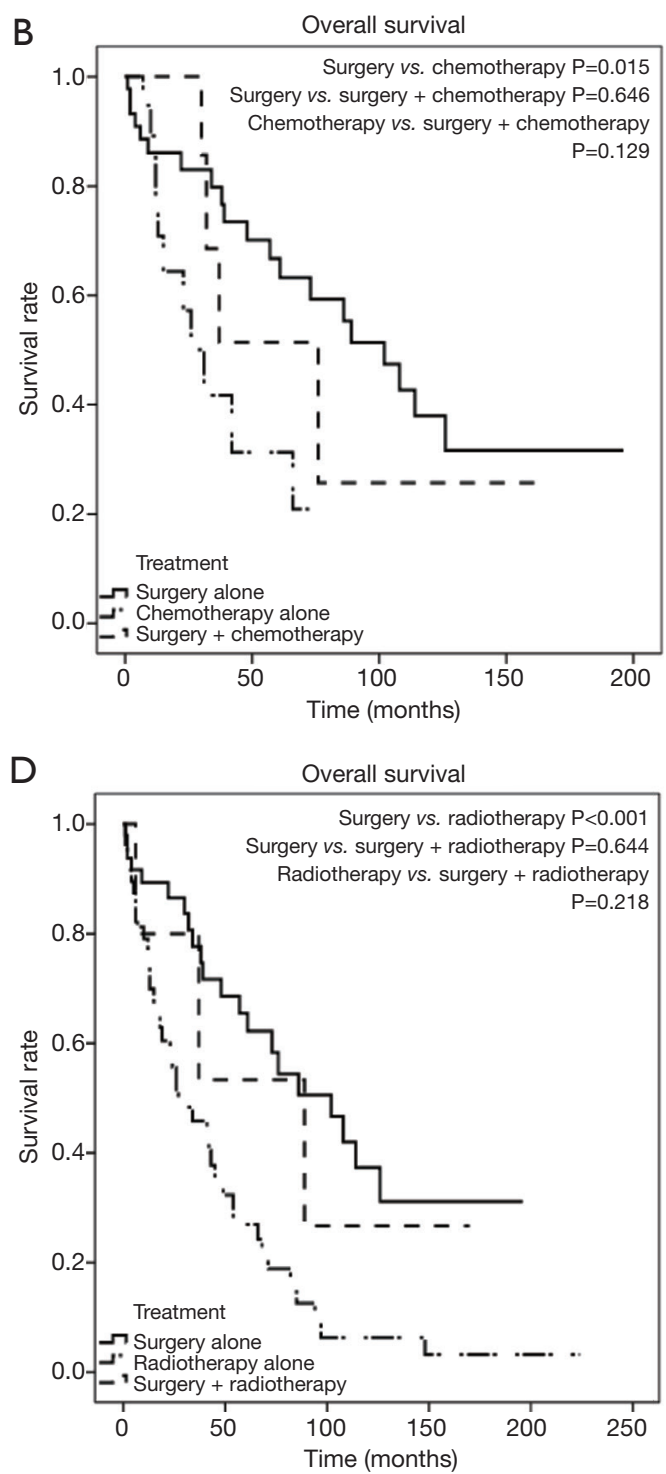

Figure 4 Kaplan-Meier analysis between different therapy modalities of patients with pulmonary papillary squamous cell carcinoma (PSCC). $(\mathrm{A}, \mathrm{B})$ Cancer-specific (A) and overall (B) survival of patients accepting surgery alone, chemotherapy alone, and surgery + chemotherapy. $(\mathrm{C}, \mathrm{D})$ Cancer-specific (C) and overall (D) survival of patients accepting surgery alone, radiotherapy alone, and surgery + radiotherapy.

occurring in 11 cases; nodal metastasis occurred in 19 cases; and distant metastases were found in 11 cases (Table 2). These data reveal the invasiveness of seemingly indolent pulmonary PSCC.

Though pulmonary PSCC exhibited its aggressiveness, our study validated another report with respect to the increased survival of PSCC compared with other types of SCCs (30). In our study, both CSS and OS were significantly longer in pulmonary PSCC (Figure 2). The improved prognosis of pulmonary PSCC compared with pulmonary SCC demonstrates the inherent heterogeneities in behavior that this variant displays. Furthermore, using the SEER database, we found an interesting trend in pulmonary cancer. There was a significant decrease for all other pulmonary cancers between 2000 and 2015 (P=0.003; Figure 1, Table 1), a finding that echoes the World Health Organization report linking such improvements to public awareness regarding smoking cessation (1). At the same 
time, however, the annual percentage change in incidence for pulmonary PSCC remained stable. Does HPV in the US population contribute to maintaining the incidence of pulmonary PSCC? Current and future exploration on etiologic factors and molecular mechanisms may reveal satisfactory answers.

As expected, for patients with pulmonary PSCC, our study demonstrated that those who underwent surgery had improved CSS and OS compared with those who did not (Figure 3). Certainly, surgical therapy is the mainstay of treatment for pulmonary cancers. On the other hand, the roles of chemotherapy and radiotherapy are vague (31-33). Conventionally, decision-making regarding the treatment of patients with PSCCs is mainly based on clinical experience and there is no standard protocol for treating these tumors. Unfortunately, in this study, the impact of chemotherapy in our cohort seems to be unsatisfactory, even in combination with surgery. Even worse, radiotherapy plays a detrimental role for both CSS and OS (Figures 3,4). Such phenomenon may partially be attributed to the late TNM stage of patients with pulmonary PSCC (Table 2). Furthermore, unsatisfactory effects of chemotherapy/radiotherapy may indicate that the occurrence of pulmonary PSCC may not be solitary and can be complicated by other diseases, which echoes one previous report (34). In addition, metastasis (from/to other parts) is another complex reason that can hinder interpretation of pulmonary tumors (4). To date, there is still no persuasive evidence explaining the ineffectiveness of chemotherapy/radiotherapy in pulmonary PSCC and future work is required to explore the relevant mechanisms. Collectively, our results suggest that surgery is the only effective treatment for pulmonary PSCC; chemotherapy or radiotherapy can be ineffective or even detrimental for survival rate.

Despite using population-based data, this study still has some limitations. First, the lack of HPV status in the SEER database hindered relevant analysis. Second, there were insufficient details concerning surgery types, drug usage, radiotherapy doses, and comorbidities to support further analyses. Third, data input under various circumstances (such as different times, locations, recorders, etc.) may introduce considerable heterogeneities. Additionally, since the study is based on a retrospective analysis, we cannot review the slides and confirm the diagnosis ourselves.

Despite the above limitations, using the SEER database still yields great benefits. The standardized database can eliminate biases caused by geographical and institutional heterogeneity during analysis. Moreover, the robust number can help researchers to draw powerful conclusions especially in the field of rare malignancies like pulmonary PSCC.

\section{Conclusions}

Our population-based evidence shows that pulmonary PSCC, as a rare cancer, has better prognosis compared with other types of pulmonary SCC. For both CSS and OSC of pulmonary PSCC, surgery is the only effective treatment; while chemotherapy has no effect and radiotherapy worsens prognosis.

\section{Acknowledgments}

The authors would like to thank the SEER database for providing open access.

\section{Footnote}

Conflicts of Interest: The authors have no conflicts of interest to declare.

Ethical Statement: The authors are accountable for all aspects of the work, ensuring that questions related to the accuracy or integrity of any part of the work are appropriately investigated and resolved. Because of the omitted patient identifiers, it was unnecessary to obtain the approval of the Institutional Review Board in this study.

\section{References}

1. Stewart BW, Wild CP. World Cancer Report 2014. International Agency for Research Cancer, World Health Organization, 2014:350-60.

2. Tajima S, Takanashi Y, Koda K. Squamous cell carcinoma of the lung with highly proliferating fibromatosislike stroma: a rare phenomenon. Int J Clin Exp Pathol 2015;8:5870-6.

3. Dulmet-Brender E, Jaubert F, Huchon G. Exophytic endobronchial epidermoid carcinoma. Cancer 1986;57:1358-64.

4. Cooper L, Hagenschneider JK, Banky S, et al. Papillary endobronchial squamous cell carcinoma. Ann Diagn Pathol 2005;9:284-8.

5. Ly V, Gupta S, Desoto F, et al. Tracheal squamous cell carcinoma treated endoscopically. J Bronchology Interv 
Pulmonol 2010;17:353-5.

6. Zhang X, Ding J, Tao X, et al. Diagnosis and treatment of cervical papillary squamous cell carcinoma with unknown depth of stromal invasion. Int J Gynaecol Obstet 2017;138:190-3.

7. Kadota K, Nitadori J, Woo KM, et al. Comprehensive pathological analyses in lung squamous cell carcinoma: single cell invasion, nuclear diameter, and tumor budding are independent prognostic factors for worse outcomes. J Thorac Oncol 2014;9:1126-39.

8. Coissard CJ, Besson G, Polette MC, et al. Prevalence of human papillomaviruses in lung carcinomas: a study of 218 cases. Mod Pathol 2005;18:1606-9.

9. Hirsch FR, Scagliotti GV, Mulshine JL, et al. Lung cancer: current therapies and new targeted treatments. Lancet 2017;389:299-311.

10. Campbell JD, Alexandrov A, Kim J, et al. Distinct patterns of somatic genome alterations in lung adenocarcinomas and squamous cell carcinomas. Nat Genet 2016;48:607-16.

11. Ferone G, Song JY, Sutherland KD, et al. SOX2 Is the Determining Oncogenic Switch in Promoting Lung Squamous Cell Carcinoma from Different Cells of Origin. Cancer Cell 2016;30:519-32.

12. Ferris RL, Blumenschein G Jr, Fayette J, et al. Nivolumab for Recurrent Squamous-Cell Carcinoma of the Head and Neck. N Engl J Med 2016;375:1856-67.

13. Oshimori N, Oristian D, Fuchs E. TGF-beta promotes heterogeneity and drug resistance in squamous cell carcinoma. Cell 2015;160:963-76.

14. Ohashi S, Miyamoto S, Kikuchi O, et al. Recent Advances From Basic and Clinical Studies of Esophageal Squamous Cell Carcinoma. Gastroenterology 2015;149:1700-15.

15. Serra A, Caltabiano R, Scalia G, et al. Papillary squamous cell carcinoma of the palatine tonsil: a rare cancer of the head and neck. Acta Otorhinolaryngol Ital 2017;37:341-5.

16. Benedetti MD, Marangi A, Bozzetti S, et al. HPV-related papillary squamous cell carcinoma of the tonsil during treatment with fingolimod. Mult Scler Relat Disord 2018;23:24-6.

17. Alotaiby F, Song F, Boyce BJ, et al. Unusual Papillary Squamous Cell Carcinoma of the Tip of Tongue Presenting in a Patient Status Post Heart Transplant. Anticancer Res 2018;38:4203-6.

18. Ferrer MJ, Estelles E, Villanueva A, et al. Papillary squamous cell carcinoma of the oropharynx. Eur Arch Otorhinolaryngol 2003;260:444-5.

19. Marques YM, Chicaro CF, Tosta M, et al. Papillary squamous cell carcinoma of the tongue. Otolaryngol Head
Neck Surg 2009;141:298-9.

20. Seiwert TY, Zuo Z, Keck MK, et al. Integrative and comparative genomic analysis of HPV-positive and HPVnegative head and neck squamous cell carcinomas. Clin Cancer Res 2015;21:632-41.

21. Sankaranarayanan R, Nene BM, Shastri SS, et al. HPV screening for cervical cancer in rural India. N Engl J Med 2009;360:1385-94.

22. Chaturvedi AK. Beyond cervical cancer: burden of other HPV-related cancers among men and women. J Adolesc Health 2010;46:S20-6.

23. Xiaofeng Q, Wei H. Papillary Squamous Cell Carcinoma of the Cheek. J Craniofac Surg 2016;27:e416.

24. Imai H, Takahashi T, Taira T, et al. Papillary squamous cell carcinoma of the trachea associated with human papillomavirus-18 infection. Intern Med 2013;52:2785-8.

25. Castellsagué X, Alemany L, Quer M, et al. HPV Involvement in Head and Neck Cancers: Comprehensive Assessment of Biomarkers in 3680 Patients. J Natl Cancer Inst 2016;108:djv403.

26. Mehrad M, Carpenter DH, Chernock RD, et al. Papillary squamous cell carcinoma of the head and neck: clinicopathologic and molecular features with special reference to human papillomavirus. Am J Surg Pathol 2013;37:1349-56.

27. Syrjanen S. Human papillomavirus (HPV) in head and neck cancer. J Clin Virol 2005;32 Suppl 1:S59-66.

28. Van Batavia J, Yamany T, Molotkov A, et al. Bladder cancers arise from distinct urothelial sub-populations. Nat Cell Biol 2014;16:982-91, 1-5.

29. Chaux A, Soares F, Rodríguez I, et al. Papillary squamous cell carcinoma, not otherwise specified (NOS) of the penis: clinicopathologic features, differential diagnosis, and outcome of 35 cases. Am J Surg Pathol 2010;34:223-30.

30. Dutta R, Husain Q, Kam D, et al. Laryngeal Papillary Squamous Cell Carcinoma: A Population-Based Analysis of Incidence and Survival. Otolaryngol Head Neck Surg 2015;153:54-9.

31. Locher C, Pourel N, Le Caer H, et al. Impact of a comprehensive geriatric assessment to manage elderly patients with locally advanced non-small-cell lung cancers: An open phase II study using concurrent cisplatin-oral vinorelbine and radiotherapy (GFPC 08-06). Lung Cancer 2018;121:25-9.

32. Stam B, van der Bijl E, van Diessen J, et al. Heart dose associated with overall survival in locally advanced NSCLC patients treated with hypofractionated chemoradiotherapy. Radiother Oncol 2017;125:62-5. 
33. Contreras JA, Lin AJ, Weiner A, et al. Cardiac dose is associated with immunosuppression and poor survival in locally advanced non-small cell lung cancer. Radiother Oncol 2018;128:498-504.

Cite this article as: Yuan Q, Sun N, Meng Z, Chen X. Pulmonary papillary squamous cell carcinoma: a populationbased analysis of incidence, treatment, and prognosis. J Thorac Dis 2019;11(10):4271-4281. doi: 10.21037/jtd.2019.09.71
34. Afridi F, Pellini Ferreira B, Geigel E. A Rare Case of Papillary Endobronchial Squamous Cell Carcinoma. Am J Respir Crit Care Med 2017;195:A6569. 